\title{
Calibration of Nonstationary Gas Sensors Based on Two- Dimensional Materials
}

\author{
Filiberto Ricciardella,* Kangho Lee, Tobias Stelz, Oliver Hartwig, Maximilian Prechtl, Mark McCrystall, \\ Niall McEvoy, and Georg S. Duesberg*
}

Cite This: ACS Omega 2020, 5, 5959-5963

Read Online

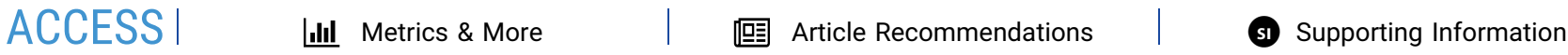

ABSTRACT: Two-dimensional materials (2DMs) have high potential in gas sensing, due to their large surface-to-volume ratio. However, most sensors based on 2DMs suffer from the lack of a steady state during gas exposure, hampering sensor calibration. Here, we demonstrate that analysis of the time differential of the signal output enables the calibration of chemiresistors based on platinum or tungsten diselenide $\left(\mathrm{PtSe}_{2}, \mathrm{WSe}_{2}\right)$ and molybdenum disulfide $\left(\mathrm{MoS}_{2}\right)$, which present nonstationary behavior. 2DMs are synthesized by thermally assisted conversion of predeposited metals on a silicon/silicon dioxide substrate and therefore are integrable with standard complementary metal-oxide semiconductor (CMOS) technology. We analyze the behavior of the sensors at room temperature toward nitrogen dioxide $\left(\mathrm{NO}_{2}\right)$ in a narrow range from 0.1 to $1 \mathrm{ppm}$. This study overcomes the problem of the absence of steady-state signals in 2DM
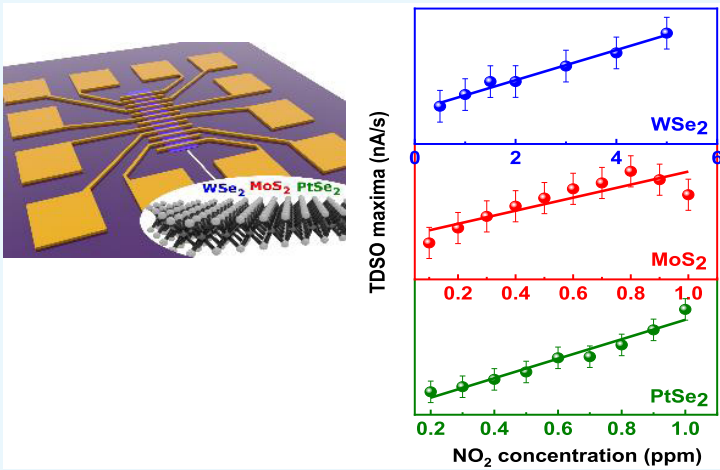
gas sensors and thus facilitates their usage in this highly important application.

\section{INTRODUCTION}

The monitoring of toxic and flammable gases at low concentration is challenging, especially in domestic and industrial environments. ${ }^{1}$ Among the waste species produced nowadays, nitrogen dioxide $\left(\mathrm{NO}_{2}\right)$ represents one of the most common exhaust gases as it is usually obtained from numerous industrial applications. This oxidizing gas can have a severe effect on human health, particularly in terms of permanent damage to the respiratory system, even at concentration levels as low as $1 \mathrm{ppm}$ (parts per million)., ${ }^{2,3}$ As such, gas sensors capable of detecting extremely low concentrations are highly sought after. ${ }^{4}$ Over the previous few decades, metal oxides $\left(\mathrm{MO}_{x}\right)$ have shown their potential as sensing materials due to their high sensitivity to pollutant gases, small size of devices, and low cost. ${ }^{1,5}$ However, it is known that $\mathrm{MO}_{x}$-based sensors only perform well when working at temperatures typically higher than $400{ }^{\circ} \mathrm{C}$, with consequent huge consumption of energy. $^{5-8}$

Sensors based on low-dimensional materials, including carbon nanotubes, graphene, and black phosphorus, have been widely investigated as promising alternatives to $\mathrm{MO}_{x}{ }^{2,9-152,9-15}$ Semiconducting two-dimensional (2D) transition metal dichalcogenides (TMDCs) have been widely investigated as promising gas-sensing materials due to their high surface-to-volume ratio, favorable surface energy levels for gas adsorption, high mobilities, and high current on/off ratios. ${ }^{16-19}$ A number of TMDCs have shown low detection limits for $\mathrm{NO}_{2}$ operating at room temperature (RT). ${ }^{19-23}$
Recently, platinum diselenide $\left(\mathrm{PtSe}_{2}\right)$ has gained huge interest for selective $\mathrm{NO}_{2}$ sensing with a low limit of detection (LOD) in the range of few $\mathrm{ppb}$ and an extremely fast response time (few seconds) when exposed to $0.1 \mathrm{ppm}^{24,25}$

However, most gas sensors based both on TMDCs and other low-dimensional materials suffer significantly from drawbacks such as the lack of a steady state during the gas exposure and slow recovery kinetics. These two hindrances can hamper the use of sensors since two characteristic parameters, the response time and the signal variation, cannot be properly determined. ${ }^{26,27}$ In previous reports, ${ }^{28-30}$ we have demonstrated that the limitations can be overcome simply by analyzing the time differential of the signal output (TDSO) instead of the output itself. In those papers, the sensing material consisted of multilayered graphene (MLG), synthesized through different routes.

Here, we demonstrate the reliability of the TDSO approach applying it to chemiresistors (CRs) based on three different TMDCs: platinum diselenide $\left(\mathrm{PtSe}_{2}\right)$, tungsten diselenide $\left(\mathrm{WSe}_{2}\right)$, and molybdenum disulfide $\left(\mathrm{MoS}_{2}\right)$. The sensors show continuously rising current during a gas exposure longer than 2

Received: December 17, 2019

Accepted: February 21, 2020

Published: March 10, 2020 
min, similar to those based on MLG. ${ }^{28-31}$ By means of TDSO, we are able to properly calibrate and compare the sensors, highlighting the promising potentialities of these materials in the field of gas sensors working at room temperature (RT).

\section{RESULTS AND DISCUSSION}

Raman and X-ray photoelectron spectroscopy (XPS) results reported in our previous publications show the stochiometric transformation of $\mathrm{Pt}, \mathrm{Mo}$, and $\mathrm{W}$ into $\mathrm{PtSe}_{2}, \mathrm{MoS}_{2}$, and $\mathrm{WSe}_{2}$, respectively, by thermally assisted conversion (TAC). ${ }^{20-22,25,32-34}$ Here, we present the application of TDSO to the devices based on TMDCs reported in those papers, in which a detailed characterization of TMDCs is presented.

Figure 1 shows the optical images and the current-voltage $(I-V)$ characteristics of the devices based on the aforemen-
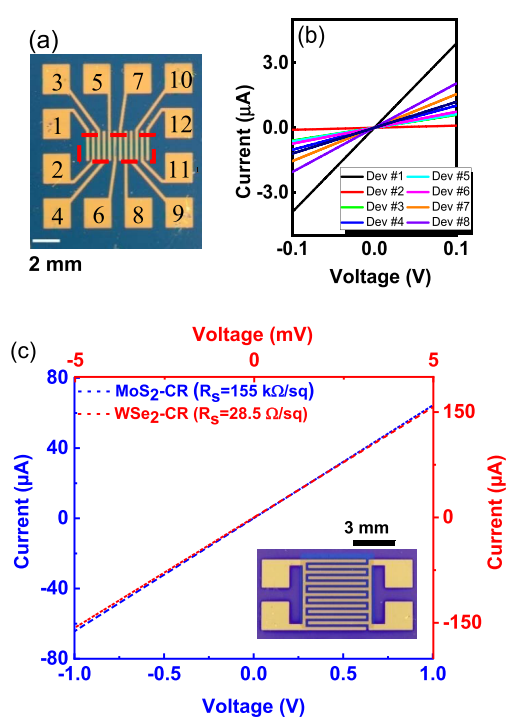

Figure 1. (a) Optical image of the chip containing the eight devices based on $\mathrm{PtSe}_{2}$ with labeled pads. The dashed red rectangle surrounds the $\mathrm{PtSe}_{2}$ film. (b) $I-V$ characteristics of the $\mathrm{PtSe}_{2}$-based resistors reported in Table S1. (c) $I-V$ characteristics of the resistors based on $\mathrm{MoS}_{2}$ (blue line) and $\mathrm{WSe}_{2}$ (red line). Inset: sketch of the $\mathrm{MoS}_{2}$ $\left(\mathrm{WSe}_{2}\right)$ sensor.

tioned TMDCs. The linearity of the $I-V$ characteristics (Figure $1 \mathrm{~b}, \mathrm{c}$ ) recorded for the investigated devices suggests that the possibility that Schottky barriers at the contacts to the $\mathrm{PtSe}_{2}, \mathrm{MoS}_{2}$, and $\mathrm{WSe}_{2}$ films can be excluded. For the $\mathrm{PtSe}_{2}-$ based devices, the values of sheet resistance $\left(R_{\mathrm{S}}\right)$ are in the range of a few hundred $\mathrm{k} \Omega / \mathrm{sq}$ (Table 1 ). The variance of the resistance values can likely be attributed to thickness variation and roughness of the sputtered Pt film at the nanoscale level.

Figure $2 \mathrm{a}$ illustrates the real-time current behavior (black line) of $\mathrm{PtSe}_{2}-\mathrm{CR} \# 1$ upon sequential exposures of $\mathrm{NO}_{2}$ (red rectangles). The signals recorded for the other tested $\mathrm{PtSe}_{2}$ based devices are shown in the Supporting Information (Figure S1).

In each exposure window, the current shows an increasing signal, never reaching the stationary state, especially for $\mathrm{NO}_{2}$ concentrations higher than $0.5 \mathrm{ppm}$. Similar results are reported elsewhere. ${ }^{28-31}$ The lack of a steady state is more evident in Figure $2 b$, where the signals recorded upon exposure to different concentrations are grouped. It can be seen that the variation of the current toward $100 \mathrm{ppb}$ of $\mathrm{NO}_{2}$ (black curve)
Table 1. Differential Sensitivity (DS) of $\mathrm{PtSe}_{2}$-Based Sensors

\begin{tabular}{cc} 
device & differential sensitivity $[\mathrm{nA} /(\mathrm{s} \cdot \mathrm{ppm})]$ \\
$\# 1$ & $0.91 \pm 0.07$ \\
$\# 2$ & $0.93 \pm 0.07$ \\
$\# 3$ & $0.82 \pm 0.11$ \\
$\# 4$ & $0.83 \pm 0.06$ \\
$\# 5$ & $0.93 \pm 0.06$ \\
$\# 6$ & $0.99 \pm 0.08$ \\
$\# 7$ & $0.88 \pm 0.04$ \\
$\# 8$ & $0.80 \pm 0.04$ \\
\hline
\end{tabular}
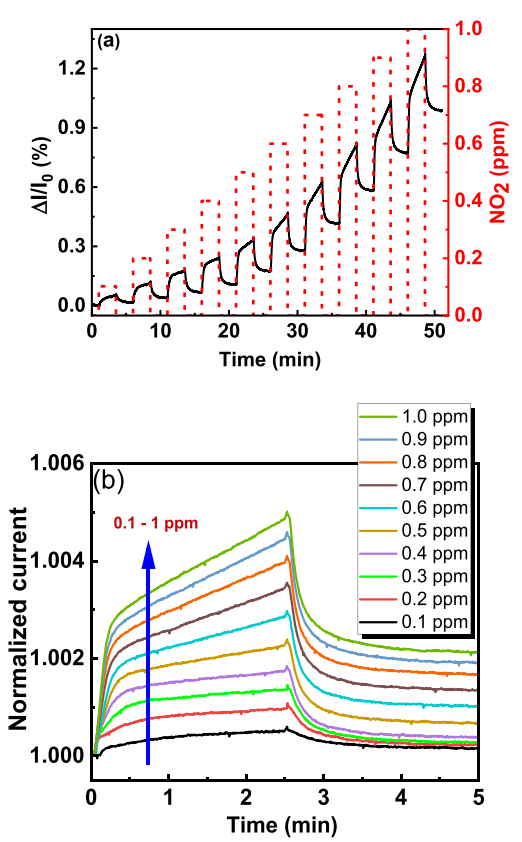

Figure 2. (a) Real-time current behavior (black curve) of $\mathrm{PtSe}_{2}-\mathrm{CR}$ \#1 during exposure to increasing concentrations of $\mathrm{NO}_{2}$ (red dashed rectangles). Dry $\mathrm{N}_{2}$ is used as a buffer gas. (b) Signals recorded upon exposure to different concentrations of $\mathrm{NO}_{2}$. The current is normalized at the value reached when each gas exposure starts.

is almost negligible compared to the others at higher concentrations.

Figure $2 \mathrm{~b}$ also shows that the higher the concentration of the injected gas, the smaller the return of the current to the initial state. Noteworthily, based on the definition of the term "recovery" as the time required to reduce the stationary signal value by $90 \%,{ }^{26}$ we need to carefully adopt this term to describe the restoration of the signal after the gas pulse. At concentrations lower than $0.5 \mathrm{ppm}$, the current value is almost restored to the initial value in $150 \mathrm{~s}$. When the sensor is exposed to the highest concentration ( $1 \mathrm{ppm})$, for instance, the current is lowered by less than $30 \%$ from the value reached when the gas is removed. These outcomes straightforwardly point out two features: first, the continuous integrating capability of the sensors and second, the difficulty in removing the adsorbed molecules in a timescale feasible for applications in environmental conditions. ${ }^{28}$ These two limitations, which commonly affect the sensors based on $2 \mathrm{D}$ materials, are mathematically overcome by the TDSO approach. ${ }^{28}$ In Figure $3 \mathrm{a}$, the method is applied to the signal recorded on device \#1. The TDSO (red curve) is overlapped upon the transients of the sensor (black line), where $I_{0}$ and $I$ represent the values of 

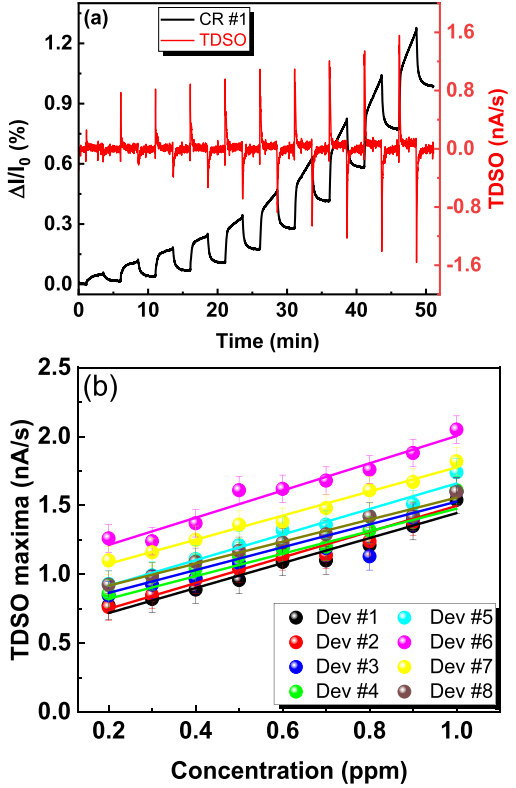

Figure 3. (a) Real-time percentage current variation (black curve) of $\mathrm{CR} \# 1$ during the exposure to increasing concentrations of $\mathrm{NO}_{2}$ and corresponding TDSO (red curve). Analogous results are obtained for the other seven devices (see the Supporting Information). (b) Maxima of the differential curves determined for the eight devices (see the Supporting Information) and plotted as a function of the $\mathrm{NO}_{2}$ concentration.

the current when the gas is injected and stopped, respectively, during each exposure. The TDSO peaks are well distinguishable for each gas pulse except at $0.1 \mathrm{ppm}$ (Figure 3a).

At that concentration, the peak is not well visible because of the small variation of the signal (Figure $2 b$ ). As the rise time of the sensor is in the range of tens of seconds, the TDSO peaks are located quite close to the position of the gas inlet $\left(t_{0}\right)$, as mathematically modeled in ref 28 (Figure S2).

The maxima of TDSO plotted as a function of the $\mathrm{NO}_{2}$ concentration in the range $0.2-1.0 \mathrm{ppm}$ (Figure $3 \mathrm{~b}$ ) display comparable behavior for the complete set of sensors, especially in terms of linearity of the fitting curves. To properly compare the performance of the sensors, the most powerful tool is differential sensitivity (DS), defined as the slope of the calibration curve. ${ }^{28}$ Further studies are ongoing to address the definition of the limit of detection through the TDSO approach. For the eight devices investigated in this paper, the values of the DS are comparable within a maximum variation of about $20 \%$ (Table 1 ).

To further prove the reliability of the TDSO approach, we tested CRs based on $\mathrm{MoS}_{2}$ and $\mathrm{WSe}_{2}$. Figure 4 reports the calibration curves obtained by applying TDSO on both sensors. For $\mathrm{MoS}_{2}$-CR (Figure 4a), the peaks were determined from the same test protocol (inset of Figure $4 \mathrm{a}$ ) used for $\mathrm{PtSe}_{2}$ CRs. WSe $\mathrm{W}_{2}-\mathrm{CR}$, instead, was revealed to be scarcely sensitive to $\mathrm{NO}_{2}$ concentrations lower than $0.4 \mathrm{ppm}$, showing no intense TDSO peaks (inset of Figure 4b). As such, we slightly modified the protocol, exposing the $\mathrm{WSe}_{2}-\mathrm{CR}$ up to $5 \mathrm{ppm}$ of $\mathrm{NO}_{2}$.

Because of the decreasing current shown by both sensors, we reported the absolute values of the TDSO peaks as a function of the $\mathrm{NO}_{2}$ concentration. The n- or p-type behavior of $\mathrm{MoS}_{2}$ is already shown in other reports, ${ }^{22,35,36}$ while we are further investigating the behavior of $\mathrm{WSe}_{2}$. Nevertheless, in the present
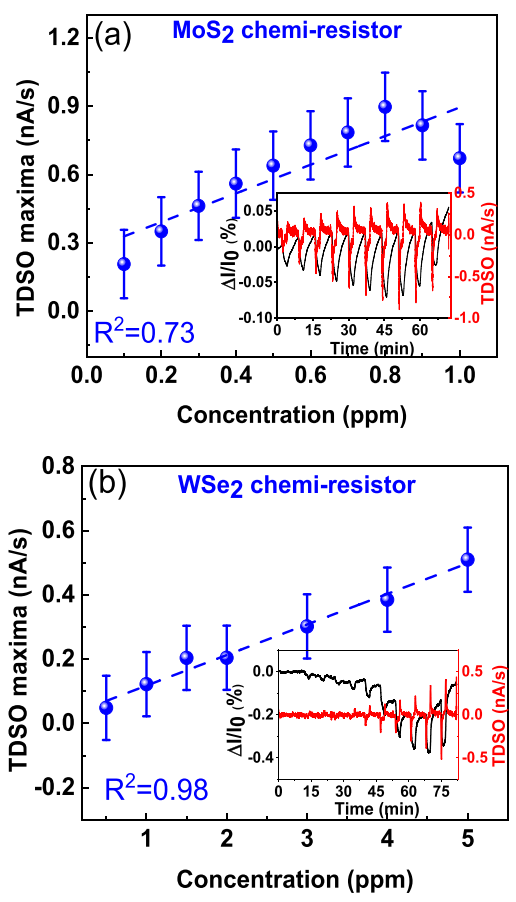

Figure 4. Calibration curves of (a) $\mathrm{MoS}_{2}$ - and (b) $\mathrm{WSe}_{2}$-based CR. The insets show the transients upon sequential $\mathrm{NO}_{2}$ exposures and TDSO, respectively, in black and red.

paper, we mostly focus on TDSO to overcome the calibration issue. The linearity of the calibration curve first confirms the reliability of the TDSO approach. The DS of $\mathrm{MoS}_{2}-\mathrm{CR}(0.6 \pm$ $0.1 \mathrm{nA} /(\mathrm{s} \cdot \mathrm{ppm}))$ indicates that TAC-grown $\mathrm{MoS}_{2}$ is slightly more sensitive than TAC-grown $\mathrm{PtSe}_{2}$ toward $\mathrm{NO}_{2}$ in the investigated range.

A fairer comparison between the results presented here and those reported in the literature can be based on the minimum detectable concentration of gas. Except for the $\mathrm{WSe}_{2}$-based $\mathrm{CR}$, the sensors presented here are able to distinguish down to a hundred $\mathrm{ppb}$ of $\mathrm{NO}_{2}$ working at RT. These findings are in close agreement with up to date results. ${ }^{16,25,37}$ By further refinement of the materials synthesis route, it is very likely that the detectable gas concentration will be lowered significantly.

\section{CONCLUSIONS}

We have analyzed the sensing properties of chemiresistors based on $\mathrm{PtSe}_{2}, \mathrm{MoS}_{2}$, and $\mathrm{WSe}_{2}$ toward $\mathrm{NO}_{2}$. The devices were able to detect concentrations of $\mathrm{NO}_{2}$ in $\mathrm{N}_{2}$ down to few hundred $\mathrm{ppb}$ at RT. We addressed the issue of a nonstationary state shown during the gas exposure by applying the TDSO approach. We definitively proved that the maxima of TDSO are uniquely and linearly correlated with the $\mathrm{NO}_{2}$ concentration in the range $0.1-1 \mathrm{ppm}$. The TDSO approach allowed us to properly calibrate the sensors based on three different TMDCs, showing the potential of these materials in the field of gas sensors operating at RT.

\section{EXPERIMENTAL SECTION}

Films of $\mathrm{PtSe}_{2}, \mathrm{MoS}_{2}$, and $\mathrm{WSe}_{2}$ were synthesized by thermally assisted conversion (TAC) of $\mathrm{Pt}, \mathrm{Mo}$, and $\mathrm{W}$, respectively. Thin layers of $\mathrm{Pt}(0.5 \mathrm{~nm})$, Mo $(10 \mathrm{~nm})$, and $\mathrm{W}(20 \mathrm{~nm})$ were sputtered onto $\mathrm{SiO}_{2}(300 \mathrm{~nm}) / \mathrm{Si}$ substrates. The samples were then loaded into a quartz tube furnace and heated at 400, 750, and $600{ }^{\circ} \mathrm{C}$, respectively. The vapors of the chalcogens were 
produced at $\sim 115^{\circ} \mathrm{C}(\mathrm{S})$ and $\sim 220{ }^{\circ} \mathrm{C}$ (Se) and diffused into the metal layers to form $\mathrm{PtSe}_{2}, \mathrm{MoS}_{2}$, and $\mathrm{WSe}_{2}$, respectively. The TAC process is described in detail in previous reports. $^{20,21,25}$

To fabricate the gas sensors, we used shadow masks to sputter Pt, Mo, and W layers only on selected areas. After the synthesis of patterned $\mathrm{PtSe}_{2}, \mathrm{MoS}_{2}$, and $\mathrm{WSe}_{2}$ by the TAC process, metal contacts $\left(\mathrm{Ni} / \mathrm{Au}\right.$ for $\mathrm{PtSe}_{2}$ or $\mathrm{WSe}_{2}, \mathrm{Ti} / \mathrm{Au}$ for $\mathrm{MoS}_{2}$ ) were deposited using hard masks to define the sensing areas. $^{21,25}$ For instance, Figure 1 illustrates the $\mathrm{PtSe}_{2}$-based CRs. Each device is about $1 \times 0.2 \mathrm{~mm}^{2}$. The electrical measurements were performed using a Keithley 2636 Source Meter Unit and a Keithley 3706 System Switch/Multimeter. We tested the gas sensors based on TMDC in a custom-made chamber with a volume of about $10 \mathrm{cL}$ and remotecontrollable mass-flow controllers (MFCs). The pressure and temperature were kept constant at 150 Torr and room temperature (RT). A constant flow of the gas mixture (100 sccm) was flushed in the chamber. We injected $10 \mathrm{ppm}$ of $\mathrm{NO}_{2}$ into the test chamber, diluting it with dry nitrogen $\left(\mathrm{N}_{2}\right)$ to achieve concentrations ranging from 0.1 to $1 \mathrm{ppm}$. Each of the ten gas pulses and subsequent recovery steps lasted $150 \mathrm{~s}$. The sensors were biased at $1 \mathrm{~V}$. The resistance of the devices upon the periodic gas exposure was simultaneously monitored.

\section{ASSOCIATED CONTENT}

\section{SI Supporting Information}

The Supporting Information is available free of charge at https://pubs.acs.org/doi/10.1021/acsomega.9b04325.

Sheet resistance $\left(R_{\mathrm{s}}\right)$ values of $\mathrm{PtSe}_{2}$-based resistors; real-time current behavior and TDSO of $\mathrm{PtSe}_{2}$-based chemiresistors (CR) upon sequential exposures of $\mathrm{NO}_{2}$; zoom on the current signal upon $\mathrm{NO}_{2}$ pulse at $1 \mathrm{ppm}$; the corresponding TDSO; signals recorded by $\mathrm{PtSe}_{2}-\mathrm{CR}$ \#1 upon exposure to different concentrations (PDF)

\section{AUTHOR INFORMATION}

\section{Corresponding Authors}

Filiberto Ricciardella - Institute for Physics, EIT 2, Bundeswehr University Munich, 85577 Neubiberg, Germany; 이이. orcid 0000-0002-9669-5649; Email: filiberto.ricciardella@ gmail.com

Georg S. Duesberg - Institute for Physics, EIT 2, Bundeswehr University Munich, 85577 Neubiberg, Germany;

Email: duesberg@unibw.de

\section{Authors}

Kangho Lee - Institute for Physics, EIT 2, Bundeswehr University Munich, 85577 Neubiberg, Germany

Tobias Stelz - Institute for Physics, EIT 2, Bundeswehr University Munich, 85577 Neubiberg, Germany

Oliver Hartwig - Institute for Physics, EIT 2, Bundeswehr University Munich, 85577 Neubiberg, Germany

Maximilian Prechtl - Institute for Physics, EIT 2, Bundeswehr University Munich, 85577 Neubiberg, Germany

Mark McCrystall - School of Physics, Trinity College Dublin, Dublin 2, Ireland

Niall McEvoy - Advanced Materials and Bioengineering Research Centre (AMBER) and School of Chemistry, Trinity College Dublin, Dublin 2, Ireland; 이이.org/0000-00015950-8755

Complete contact information is available at: https://pubs.acs.org/10.1021/acsomega.9b04325

\section{Author Contributions}

F.R conceived the idea, analyzed the data, and wrote the manuscript. K.L. performed the sensing measurements. T.S. assisted in plotting the data and formatted the manuscript. O.H. and M.P. performed the material characterization. M.M. and N.M. synthesized the material, and G.S.D. supervised the manuscript. All authors provided their own contribution in writing the manuscript.

\section{Notes}

The authors declare no competing financial interest.

\section{ACKNOWLEDGMENTS}

This project has received funding from the European Union's Horizon 2020 under grant agreement no. 785219 (Graphene Flagship). This project was supported with funds from the German Federal Ministry for Education and Research (BMBF) in the context of the national security research programme. Furthermore, the authors would like to thank Riley Gatensby (AMBER and School of Chemistry, Trinity College Dublin) for the fabrication of the devices.

\section{REFERENCES}

(1) Anukunprasert, T.; Saiwan, C.; Traversa, E. The Development of Gas Sensor for Carbon Monoxide Monitoring Using Nanostructure of Nb-TiO2. Sci. Technol. Adv. Mater. 2005, 6, 359-363.

(2) Wu, E.; Xie, Y.; Yuan, B.; Zhang, H.; Hu, X.; Liu, J.; Zhang, D. Ultrasensitive and Fully Reversible $\mathrm{NO}_{2}$ Gas Sensing Based on PType MoTe2 under Ultraviolet Illumination. ACS Sens. 2018, 3, $1719-1726$.

(3) Ricciardella, F. From graphene to graphene-based gas sensors operating in environmental conditions. Ph.D. Thesis, Università degli Studi di Napoli Federico II, 2015.

(4) Baron, R.; Saffell, J. Amperometric Gas Sensors as a Low Cost Emerging Technology Platform for Air Quality Monitoring Applications: A Review. ACS Sens. 2017, 2, 1553-1566.

(5) Liu, Y.; Parisi, J.; Sun, X.; Lei, Y. Solid-State Gas Sensors for High Temperature Applications - a Review. J. Mater. Chem. A 2014, 2, 9919-9943.

(6) Kim, H.; Lee, J. Sensors and Actuators B : Chemical Highly Sensitive and Selective Gas Sensors Using p-Type Oxide Semiconductors : Overview. Sens. Actuators, B 2014, 192, 607-627.

(7) Dey, A. Semiconductor Metal Oxide Gas Sensors: A Review. Mater. Sci. Eng. B 2018, 229, 206-217.

(8) Arshak, K.; Moore, E.; Lyons, G. M.; Harris, J.; Clifford, S. A Review of Gas Sensors Employed in Electronic Nose Applications. Sens. Rev. 2004, 24, 181-198.

(9) Nag, A.; Mitra, A.; Chandra, S. Sensors and Actuators A: Physical Graphene and Its Sensor-Based Applications: A Review. Sens. Actuators, A 2018, 270, 177-194.

(10) Llobet, E. Gas Sensors Using Carbon Nanomaterials: A Review. Sens. Actuators, B 2013, 179, 32-45.

(11) Iqbal, N.; Afzal, A.; Cioffi, N.; Sabbatini, L.; Torsi, L. NOx Sensing One- and Two-Dimensional Carbon Nanostructures and Nanohybrids: Progress and Perspectives. Sens. Actuators, B 2013, 181, $9-21$.

(12) Abbas, A. N.; Köpf, M.; Ma, Y.; Nilges, T.; Cong, S.; Chen, L.; Zhou, C.; Aroonyadet, N.; Liu, B. Black Phosphorus Gas Sensors. ACS Nano 2015, 9, 5618-5624.

(13) Donarelli, M.; Prezioso, S.; Perrozzi, F.; Bisti, F.; Nardone, M.; Giancaterini, L.; Cantalini, C.; Ottaviano, L. Response to $\mathrm{NO}_{2}$ and Other Gases of Resistive Chemically Exfoliated $\mathrm{MoS}_{2}$-Based Gas Sensors. Sens. Actuators, B 2015, 602-613. 
(14) Kannan, P. K.; Late, D. J.; Morgan, H.; Rout, C. S. Recent Developments in 2D Layered Inorganic Nanomaterials for Sensing. Nanoscale 2015, 7, 13293-13312.

(15) Jaiswal, J.; Sanger, A.; Tiwari, P.; Chandra, R. $\mathrm{MoS}_{2}$ Hybrid Heterostructure Thin Film Decorated with CdTe Quantum Dots for Room Temperature NO2 Gas Sensor. Sens. Actuators, B 2020, 305, No. 127437.

(16) Mao, S.; Chang, J.; Pu, H.; Lu, G.; He, Q.; Zhang, H.; Chen, J. Two-Dimensional Nanomaterial-Based Field-Effect Transistors for Chemical and Biological Sensing. Chem. Soc. Rev. 2017, 46, 68726904.

(17) Yang, W.; Gan, L.; Li, H.; Zhai, T. Two-dimensional layered nanomaterials for gas-sensing applications. Inorg. Chem. Front. 2016, 3, 433-451

(18) Zhao, Y.; Qiao, J.; Yu, Z.; Yu, P.; Xu, K.; Lau, S. P.; Zhou, W.; Liu, Z.; Wang, X.; Ji, W.; et al. High-Electron-Mobility and Air-Stable 2D Layered PtSe $e_{2}$ FETs. Adv. Mater. 2017, 29, No. 1604230.

(19) Anichini, C.; Czepa, W.; Pakulski, D.; Aliprandi, A.; Ciesielski, A.; Samorì, P. Chemical Sensing with 2D Materials. Chem. Soc. Rev. 2018, 47, 4860-4908.

(20) Gatensby, R.; Hallam, T.; Lee, K.; McEvoy, N.; Duesberg, G. S. Investigations of Vapour-Phase Deposited Transition Metal Dichalcogenide Films for Future Electronic Applications. Solid. State. Electron. 2016, 125, 39-51.

(21) Gatensby, R.; McEvoy, N.; Lee, K.; Hallam, T.; Berner, N. C.; Rezvani, E.; Winters, S.; O'Brien, M.; Duesberg, G. S. Controlled Synthesis of Transition Metal Dichalcogenide Thin Films for Electronic Applications. Appl. Surf. Sci. 2014, 297, 139-146.

(22) Lee, K.; Gatensby, R.; McEvoy, N.; Hallam, T.; Duesberg, G. S. High-Performance Sensors Based on Molybdenum Disulfide Thin Films. Adv. Mater. 2013, 25, 6699-6702.

(23) Lee, E.; Yoon, Y. S.; Kim, D. J. Two-Dimensional Transition Metal Dichalcogenides and Metal Oxide Hybrids for Gas Sensing. ACS Sens. 2018, 3, 2045-2060.

(24) Sajjad, M.; Montes, E.; Singh, N.; Schwingenschlögl, U. Superior Gas Sensing Properties of Monolayer PtSe 2. Adv. Mater. Interfaces 2017, 4, No. 1600911.

(25) Yim, C.; Lee, K.; McEvoy, N.; O’Brien, M.; Riazimehr, S.; Berner, N. C.; Cullen, C. P.; Kotakoski, J.; Meyer, J. C.; Lemme, M. C.; et al. High-Performance Hybrid Electronic Devices from Layered $\mathrm{PtSe}_{2}$ Films Grown at Low Temperature. ACS Nano 2016, 10, 95509558

(26) Nomani, M. W. K.; Shishir, R.; Qazi, M.; Diwan, D.; Shields, V. B.; Spencer, M. G.; Tompa, G. S.; Sbrockey, N. M.; Koley, G. Highly Sensitive and Selective Detection of NO2using Epitaxial Graphene on 6H-SiC. Sens. Actuators, B 2010, 150, 301-307.

(27) Yoon, H. J.; Jun, D. H.; Yang, J. H.; Zhou, Z.; Yang, S. S.; Cheng, M. M. C. Carbon Dioxide Gas Sensor Using a Graphene Sheet. Sens. Actuators, B 2011, 157, 310-313.

(28) Ricciardella, F.; Polichetti, T.; Vollebregt, S.; Alfano, B.; Massera, E.; Sarro, L. Analysis of a Calibration Method for NonStationary CVD Multi-Layered Graphene-Based Gas Sensors. Nanotechnology 2019, 30, No. 38.

(29) Ricciardella, F.; Massera, E.; Polichetti, T.; Miglietta, M. L.; Di Francia, G. A Calibrated Graphene-Based Chemi-Sensor for Sub Parts-per-Million $\mathrm{NO}_{2}$ Detection Operating at Room Temperature. Appl. Phys. Lett. 2014, 104, No. 183502.

(30) Ricciardella, F.; Vollebregt, S.; Polichetti, T.; Alfano, B.; Massera, E.; Sarro, P. M. In An Innovative Approach to Overcome Saturation and Recovery Issues of CVD Graphene-Based Gas Sensors, Proceedings of IEEE Sensors, 2017.

(31) Ricciardella, F.; Vollebregt, S.; Polichetti, T.; Miscuglio, M.; Alfano, B.; Miglietta, M. L.; Massera, E.; Di Francia, G.; Sarro, P. M. Effects of Graphene Defects on Gas Sensing Properties towards NO2 Detection. Nanoscale 2017, 9, 6085-6093.

(32) Zhang, S.; Dong, N.; McEvoy, N.; OBrien, M.; Winters, S.; Berner, N. C.; Yim, C.; Li, Y.; Zhang, X.; Chen, Z.; et al. Direct Observation of Degenerate Two-Photon Absorption and Its
Saturation in $\mathrm{WS}_{2}$ and $\mathrm{MoS}_{2}$ Monolayer and Few-Layer Films. ACS Nano 2015, 9, 7142-7150.

(33) O’Brien, M.; McEvoy, N.; Motta, C.; Zheng, J. Y.; Berner, N. C.; Kotakoski, J.; Elibol, K.; Pennycook, T. J.; Meyer, J. C.; Yim, C.; et al. Raman Characterization of Platinum Diselenide Thin Films. 2D Mater. 2016, 3, No. 021004.

(34) Kim, W. Y.; Kim, H. J.; Hallam, T.; McEvoy, N.; Gatensby, R.; Nerl, H. C.; O’Neill, K.; Siris, R.; Kim, G. T.; Duesberg, G. S. FieldDependent Electrical and Thermal Transport in Polycrystalline $\mathrm{WSe}_{2}$. Adv. Mater. Interfaces 2018, 5, No. 1701161.

(35) Zhang, Y. I.; Zhang, L.; Zhou, C. Graphene and Related Applications. Acc. Chem. Res. 2013, 46, 2329-2339.

(36) Hallam, T.; Monaghan, S.; Gity, F.; Ansari, L.; Schmidt, M.; Downing, C.; Cullen, C. P.; Nicolosi, V.; Hurley, P. K.; Duesberg, G. S. Rhenium-Doped $\mathrm{MoS}_{2}$ Films. Appl. Phys. Lett. 2017, 111, No. 203101.

(37) Donarelli, M.; Ottaviano, L. 2D Materials for Gas Sensing Applications: A Review on Graphene Oxide, $\mathrm{MoS}_{2}, \mathrm{WS}_{2}$ and Phosphorene. Sensors 2018, 18, 3638-3683. 